\title{
Hyperplastic and atrophic changes in the genital tract of a female cat following repeated treatment with deslorelin acetate - a case report
}

\author{
Gabriele Marino ${ }^{1}$, Cecilia Vullo ${ }^{2}$, Stefania Di Giorgio ${ }^{1}$, Antonina Zanghì ${ }^{1}$, \\ Giuseppe Catone ${ }^{1}$, Alessandra Sfacteria ${ }^{1}$
}

University of Messina ${ }^{1}$ Department of Veterinary Sciences, ${ }^{2}$ Department of Chemical, Biological, Pharmaceutical and Environmental Sciences, Messina, Italy

Received December 19, 2020

Accepted May 26, 2021

\begin{abstract}
This study aimed to investigate the morphological patterns of the genital tract after long-term treatment of deslorelin acetate in a female cat, a gonadotropin-releasing hormone agonist currently used in adult cats to obtain transient oestrus suppression. A 1-year-old Chartreux female cat was treated with $4.7 \mathrm{mg}$ deslorelin acetate to suppress oestrus manifestations. The treatment was repeated for a total of $\times 3$ every 2 years. After 8 years, the female cat came into oestrus again, but she was no more implanted, and an ovariohysterectomy was performed. Before surgery, an ultrasound examination was performed that showed a normal uterus and the presence of about 5 follicles in ovaries. Concentrations of oestradiol, progesterone, and vaginal smears were compatible with oestrus. During surgery, a very short ovarian pedicle was observed yet neither uterus nor ovaries presented appreciable alterations. At histology, the ovaries presented a juvenile appearance with numerous primordial and periovulatory follicles. The uterus showed marked endometrial hyperplasia with polypoid projection and atrophic myometrium. Based on this case report, deslorelin acetate is a powerful drug able to preserve ovarian function. However, the suppression of gonadotrophin, especially for a long period, has a detrimental atrophic effect on the target organs during treatment and, on the opposite, hyperplastic changes may occur after the restoring of normal cyclicity.
\end{abstract}

Feline, gonadotropin-releasing hormone agonist, polyp, uterus, fertility, histology

Deslorelin acetate is a synthetic agonist of gonadotropin-releasing hormone currently used to induce temporary sterility in sexually mature dogs and cats. Munson et al. (2001) reported for the first time the use of $6 \mathrm{mg}$ deslorelin acetate as a contraceptive in domestic cats. Three parallel studies were published in 2012, evaluating the $4.7 \mathrm{mg}$ (Ackermann et al. 2012; Pisu and Romagnoli 2012) and the $9.4 \mathrm{mg}$ deslorelin acetate implant (Toydemir et al. 2012), respectively in 10, 20 and 21 adult female cats. In the first study, oestrus was pharmacologically induced 10 days after the removal of the implant maintained in situ for 90 days; after further 6 days the queens were ovariohysterectomized and the oviducts were flushed; each queen had $13.1 \pm 5.5$ corpora lutea and produced $8.1 \pm 5.5$ viable oocytes (Ackermann et al. 2012). In the second study, the reversibility of the treatment was assessed by oestrus manifestations that occurred $22 \pm 4$ months after the implant; but most cats were still in anoestrus at the end of the study (Pisu and Romagnoli 2012). In the third study, reversibility was only observed in a queen 16.5 months after the implant by faecal oestradiol assay (Toydemir et al. 2012). In 2013, a new study on 20 queens implanted with $4.7 \mathrm{mg}$ deslorelin acetate recorded a duration of efficacy varying between 16 and 37 months; but two queens died during the treatment for other causes and, in another queen, the implant was still effective at the end of the study (GoerickePesch et al. 2013). Oestradiol and progesterone concentrations were not significantly different comparing pre- and post-treatment values. Seven out of 8 female cats, tested for post-treatment fertility, were immediately pregnant. One cat failed to get pregnant

Address for correspondence:

Gabriele Marino

Department of Veterinary Sciences, University of Messina

Viale Giovanni Palatucci, Polo Universitario SS Annunziata

98168 Messina, Italy

Phone: +3909667960

E-mail: marinog@unime.it

http://actavet.vfu.cz/ 
in the first oestrus and was mated in the two subsequent oestrus cycles until pregnancy occurred. All cats gave birth naturally and spontaneously; the litter size ranged from 1 to 5 kittens (Goericke-Pesch et al. 2013). Recently, Furthner et al. (2020) reported the reproductive data of female cats treated with subcutaneous implants of $4.7 \mathrm{mg}$ deslorelin acetate, collected from questionnaires sent to cat breeders. The duration of the efficacy of the implant, recorded in 26 cats, was $16.0 \pm 5.7$ months. Among these, 12 queens produced 3-7 kittens per litter. The literature review on deslorelin implant in female cats seems to indicate that after a single treatment, oestrus is suppressed for about 16 months and fertility may be restored with a satisfactory litter production. However, some cats required more post-treatment oestruses to be pregnant (Goericke-Pesch et al. 2013; Furthner et al. 2020). In this report, the clinical and pathological findings of a female cat that came into oestrus again after repeated implants are reported and discussed.

\section{Case description}

A Chartreux female cat at the age of 1 year was implanted with deslorelin $4.7 \mathrm{mg}$ (Suprelorin, Virbac, Carros, France) to suppress oestrus manifestations. The efficacy of the treatment lasts about 24 months. At the onset of oestrus vocalization, the queen was implanted again, and a third and fourth time, always at 2-year intervals. During the overall period of treatment, the cat was fed commercial food for neutered cats and no significant health problems were registered. After 8 years, the female cat came into oestrus again but at that time, she was treated no more. The first oestrus lasted 9 days and, the cat came into oestrus again after an inter-oestrus of 4 days. The signs observed by the owner were typical (tail deflection, spinal flexion, rubbing or rolling, vaginal discharge, vocalization, treading of the hind legs, body and tail tremor and rigidity, clawing and scratching). At this time, she was submitted to the Veterinary Teaching Hospital of the University of Messina for definitive desexing. The cat was clinically healthy, showed a moderate stress leukogram (neutrophils $1.41 \mathrm{~K} / \mu \mathrm{l}$, lymphocytes $0.91 \mathrm{~K} / \mu \mathrm{l}$ ) and decreased blood urea nitrogen $(12 \mathrm{mg} / \mathrm{dl})$. The ultrasound examination revealed a normally thin uterus and the presence of about 5 follicles of $3 \mathrm{~mm}$ in diameter at ovaries. At vaginal cytology, slides showed a clear background, no cellular debris, many superficial anuclear keratinized cells and few intermediate cells. Progesterone (P4) level was basal $(0.8 \mathrm{ng} / \mathrm{ml})$ and oestradiol 173 (E2) level was increased $(30 \mathrm{pg} / \mathrm{ml})$. The queen was premedicated with an intramuscular injection of $5 \mu \mathrm{g} / \mathrm{kg}$ dexmedetomidine (Dexdomitor; Orion Pharma, Milan, Italy) and $0.2 \mathrm{mg} / \mathrm{kg}$ methadone (Semfortan; Dechra, Turin, Italy) mixed in the same syringe. Twenty minutes after the premedication, a catheter was aseptically placed into the cephalic vein and anaesthesia was induced with IV propofol (Propovet; Zoetis, Rome, Italy) to effect before intubation. Anaesthesia was maintained with isoflurane (Isoflo; Zoetis, Rome, Italy) in $100 \%$ oxygen. The cat was positioned in dorsal recumbency and a median incision was made through the linea alba. After visualizing the left uterine horn and the ovary, the suspensory ligament was torn with the finger. A Rochester-Carmalt haemostatic forceps was placed on the pedicle proximally to the ovary and distally to it and the Sonicision cordless ultrasonic dissection device (Medtronic, Milan, Italy) was used to dissect the pedicle between the forceps and the ovary. The same method was applied for the contralateral ovary and the body of the uterus. The ovarian pedicles were very short, and hard to exteriorize. After the surgery, Cefovecin (Convenia, Zoetis, Rome, Italy) was given at $0.5 \mathrm{ml}$ (SC) and Meloxicam (Metacam, Boehringer Ingelheim, Milan, Italy) $0.2 \mathrm{ml}$ (IV). Grossly, the uterus and ovaries did not present appreciable alterations. Representative specimens were fixed in $10 \%$ formalin. All tissues were embedded in paraffin, cut and stained with haematoxylin and eosin and Masson's trichrome. At the ovaries, numerous primordial follicles were seen in the peripheral ovarian cortex, many 
developing and preovulatory follicles, the last with blood-filled capillaries in the theca interna layer (Plate XIV, Fig. 1). No luteal tissue was present. In the ovarian stroma, between the ovarian antral follicles, numerous aggregates of interstitial cells were present and appeared round to polyhedral with vacuolated, likely lipid-laden, cytoplasm. Also, the blood vessels of the ovarian medulla and especially the arterioles were ectasic and blood-filled as well as the arterioles and capillaries of the ovarian cortical stroma. The uterus showed marked hyperplasia of the endometrium with irregular thickening and mild cystic dilatation of the endometrial glands that assumed an inhomogeneous course. However, in some areas, the endometrium appeared atrophic (Plate XIV, Fig. 2). Focally, on the cross section, the endometrium developed broad base polypoid structures protruding in the dilated lumen (Plate XV, Fig. 3). These structures, made up of endometrial stroma and cystic glands, showed extensive haemorrhagic areas. The endometrial thickening was due to the increased number and size of glands with a marked reduction of the endometrial stroma. The luminal epithelium was lined by tall cuboidal cells arranged in a single layer. The myometrium appeared extremely thinner in some areas, quite atrophic for most of the transversal section of the uterine horn.

\section{Discussion}

Medical contraception in cats is a topic of great interest; its use is advocated as an alternative to surgery to desex stray cats and to control colony overpopulation. Moreover, there is a growing clinical demand from breeders looking for a safe, temporary contraceptive solution for breeding management. Deslorelin acetate implant is a product licensed for healthy, entire, sexually mature male dogs capable of provoking transitory infertility. In cats, it has been successfully employed and showed a long-lasting effect of about 16 months (Toydemir et al. 2012; Furthner et al. 2020), compared to the effects of 6 or fewer months in dogs. Despite the prolonged infertility temporal window, deslorelin acetate has a transitory effect and fertility is restored with a satisfactory litter production reported after a single treatment (Goericke-Pesch et al. 2013). This study has investigated the effects on the genital tract after repeated treatments of deslorelin acetate in a female cat. Clinically, she came into oestrus again after 24 months from the last implant, showing typical behaviour and a regular inter-oestrus. No alteration was suspected until the histological examination of the genital tract was done, except for a very short ovarian pedicle. The microscopic findings of the ovaries confirmed the functional ovarian activity (follicular phase of oestrus cycle) but, interestingly, the ovarian parenchyma maintained a juvenile appearance with numerous primordial follicles, as reported after a single implant (Toydemir et al. 2012). Deslorelin acetate, suppressing gonadotrophin production, seems capable of preserving the ovarian reserve without causing degenerative changes at the ovarian level. At the uterine level, instead, the coexistence of hypertrophic and atrophic changes was found in the endometrium and myometrium. The uterus and vagina, being the main sex hormone target organs, may develop mild to severe atrophic changes during a long pituitary suppression (Marino et al. 2014; Marino et al. 2019). However, at the end of the treatment, most of the changes should be reversible. Atypical endometrial polypoid hyperplasia has been documented for the first time in the cat and interpreted as a tumultuous effort to proliferate under sex steroid stimulation during at least two consecutive anovulatory heats of the cat. A similar observation was done by Theise (2016), reporting cystic endometrial hyperplasia associated with anovulatory heats (follicular cysts) in a female dog implanted with $9.4 \mathrm{mg}$ deslorelin acetate. The uterus and probably all the sex hormone target organs may require time to restore their full functionality which may affect full reversibility of the treatment especially for long and repeated treatments. 


\section{References}

Ackermann CL, Volpato R, Destro FC, Trevisol E, Ruas Sousa N, Guaitolini CRF, Derussi AAP, Rascado TS, Lopes MD 2012: Ovarian activity reversibility after the use of deslorelin acetate as a short-term contraceptive in domestic queens. Theriogenology 78: 817-822

Furthner E, Roos J, Niewiadomska Z, Maenhoudt C, Fontbonne A 2020: Contraceptive implants used by cat breeders in France: a study of 140 purebred cats. J Feline Med Surg 22: 984-992

Goericke-Pesch S, Georgiev P, Atanasov A, Albouy M, Navarro C, Wehrend A 2013: Treatment of queens in estrus and after estrus with a GnRH-agonist implant containing $4.7 \mathrm{mg}$ deslorelin; hormonal response, duration of efficacy, and reversibility. Theriogenology 79: 640-646

Marino G, Rizzo S, Quartuccio M, Macrì F, Pagano G, Taormina A, Cristarella S, Zanghì A 2014: Deslorelin implants in pre-pubertal female dogs: short- and long-term effects on the genital tract. Reprod Dom Anim 49: 297-301

Marino G, Quartuccio M, Sfacteria A, Cristarella S, Zanghì A 2019: Complete vaginal stenosis and hematocolpus in two bitches with a history of GnRH treatment to postpone puberty. J Hellenic Vet Med Soc 70: 1905-1910

Munson L, Bauman J E, Asa CS, Jöchle W, Trigg TE 2001: Efficacy of the GnRH analogue deslorelin for suppression of oestrous cycles in cats. J Reprod Fertil Suppl. 57: 269-273

Pisu MC, Romagnoli S 2012: Application of a single deslorelin implant in cats. Veterinaria 26: 9-15

Theise B 2016: Cystic endometrial hyperplasia and leiomyoma following perennial treatment of a bitch with Deslorelin implant. Kleintierpraxis 61: 614-621

Toydemir TSF, Kılıçarslan MR, Olgaç V 2012: Effects of the GnRH analogue deslorelin implants on reproduction in female domestic cats. Theriogenology 77: 662-674 
Plate XIV

Marino G. et al.: Hyperplastic... pp. 207-210

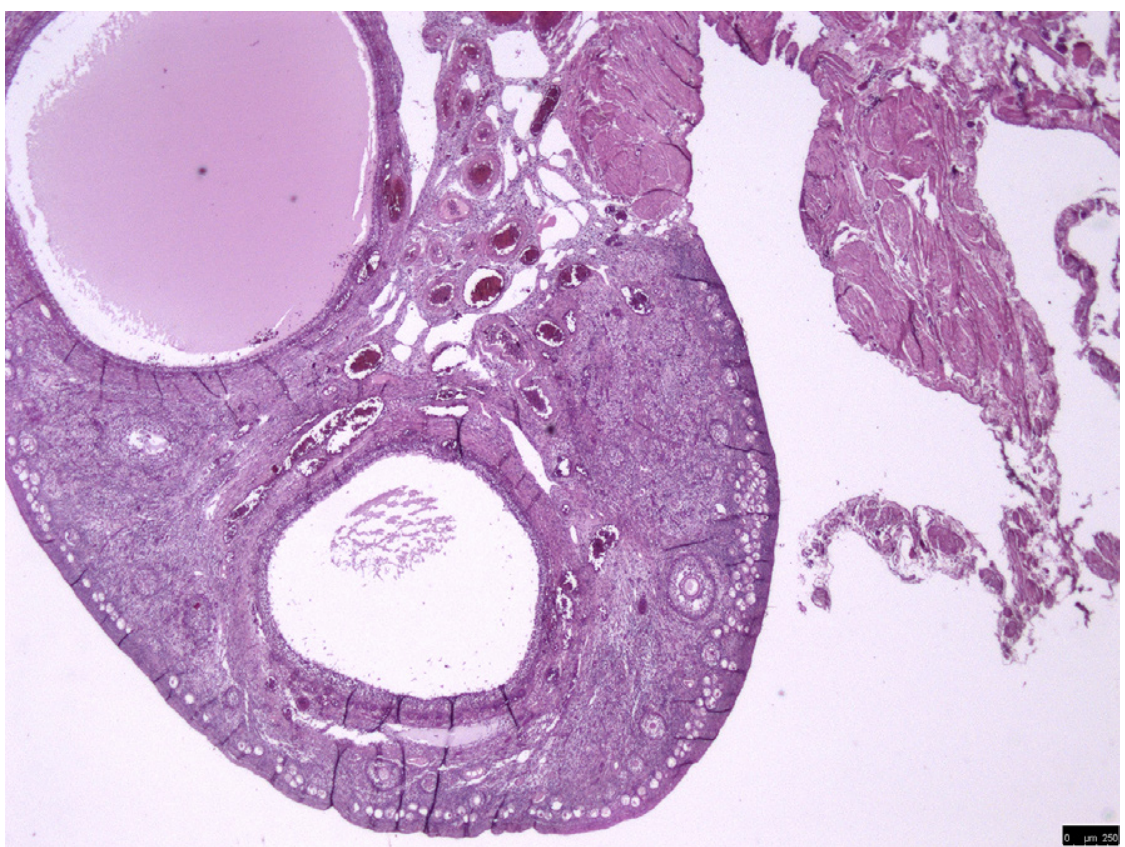

Fig. 1. The ovary with large periovulatory follicles and numerous primordial follicles in the cortex. Haematoxylin and eosin, scale bar: $250 \mu \mathrm{m}$.

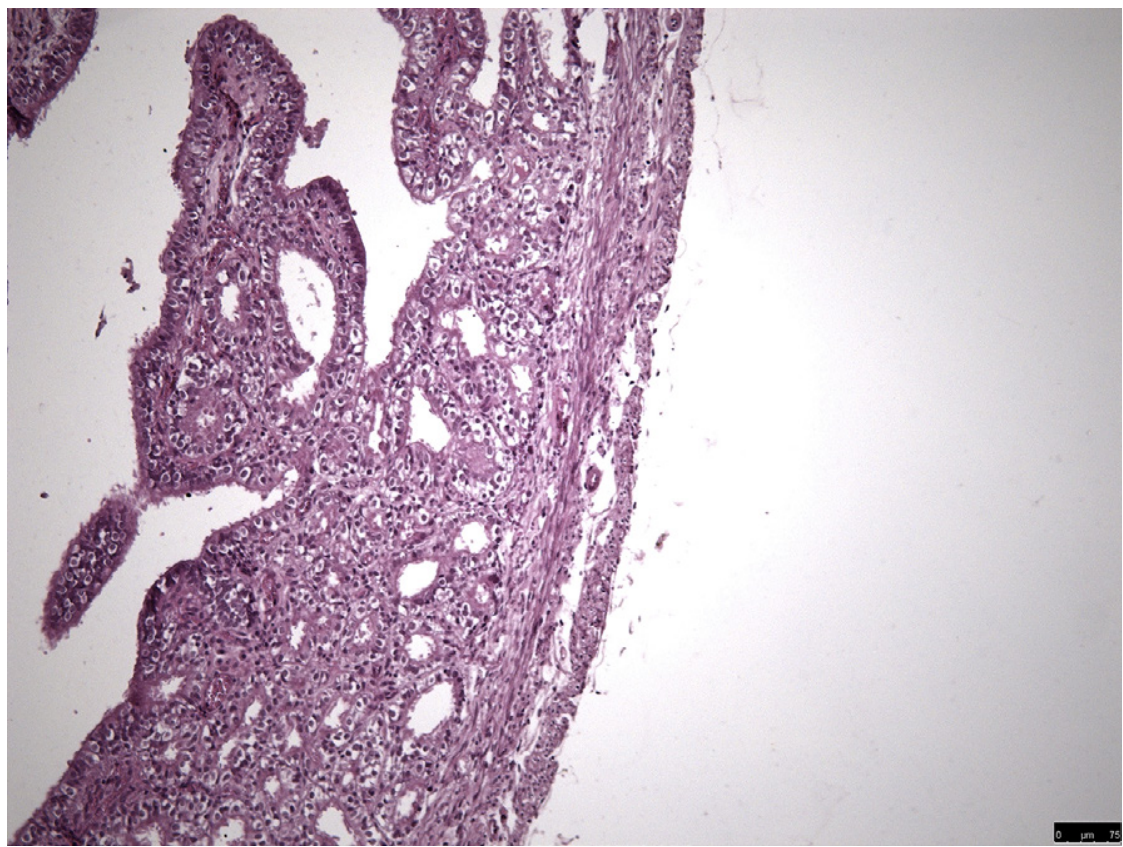

Fig. 2. A slight proliferation of endometrial glands in the endometrium with a visible thin myometrium. Haematoxylin and eosin, scale bar: $75 \mu \mathrm{m}$. 


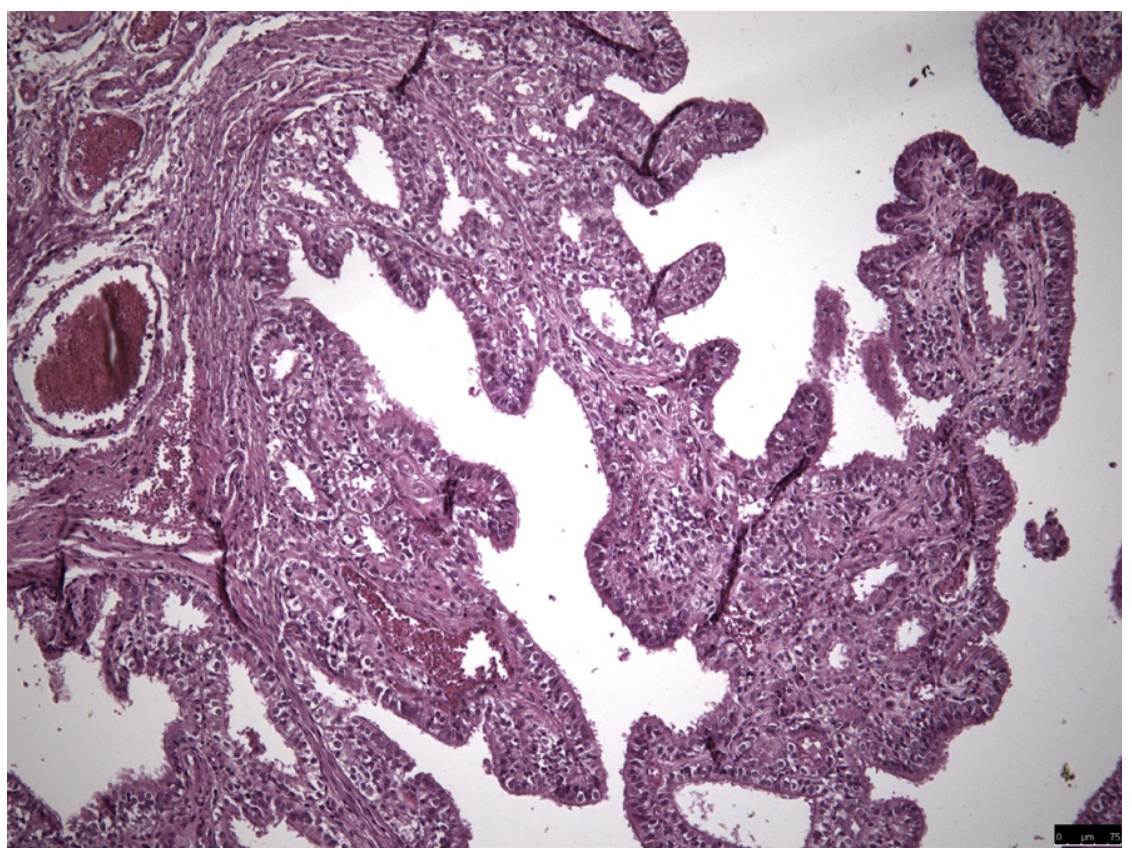

Fig. 3. The endometrium with polypoid projections in the lumen. Haematoxylin and eosin, scale bar: $75 \mu \mathrm{m}$. 